EXTENDED REPORT

\title{
Case-controlled clinical and histopathological study of conjunctivochalasis
}

\author{
I C Francis, D G Chan, P Kim, G Wilcsek, M Filipic, J Yong, M T Coroneo
}

Br J Ophthalmol 2005;89:302-305. doi: 10.1136/bjo.2004.051144

See end of article for authors' affiliations .....................

Correspondence to: Dr lan C Francis, Suite 12, Chatswood Grove, 12-14 Malvern Avenue,

Chatswood, 2067, NSW, Australia; if@student.unsw. edu.au

Accepted for publication 1 August 2004
Background/aims: Conjunctivochalasis, a secondary cause of the watery eye, is frequently seen in the older age group as an elevation of the bulbar conjunctiva lying along the lateral or central lower lid margin. A prospective, interventional, case-controlled clinical and histopathological study was conducted. The relevant features of 18 patients (29 eyes) who had their conjunctivochalasis resected as part of the surgical management of their watery eye syndrome were examined. In the control group, tissue was obtained from an age matched series of 24 normal subjects undergoing routine cataract surgery.

Methods: 24 controls ( 24 specimens) and 18 patients ( 29 specimens) had conjunctival strip biopsies, taken from the usual lid margin level bulbar conjunctiva in line with the inferior limbus (controls), and the clinically apparent conjunctivochalasis (patients). These were submitted for histological study.

Results: 23 of 24 control sections demonstrated normal conjunctival variation. Four of 29 patient specimens demonstrated a chronic non-granulomatous conjunctivitis, while three eyes of the patient group (two patients) demonstrated features of elastosis. Of the four patients who had the inflammatory infiltrates, three had functional nasolacrimal duct obstructions (FNLDOs) and one had a primary acquired nasolacrimal duct obstruction (PANDO). Of the two patients who had elastosis, one had an FNLDO and the other had normal lacrimal drainage and was Jones 1 positive.

Conclusion: Six of 18 patients - that is, seven of 29 specimens of conjunctivochalasis demonstrated signs of elastosis or of chronic non-granulomatous inflammation. Clinically, patients had a spectrum of aetiologies of their watery eye syndrome.
C onjunctivochalasis was first recognised by Braunschweig in 1921 when reported in the German literature. ${ }^{1}$ It reached the American literature in 1942 when described by Hughes, ${ }^{2}$ and was noted by Duke-Elder ${ }^{3}$ as "conjunctival hyperplasia which may require surgical removal or reduction by electrocoagulation." $\mathrm{Liu}^{4}$ described it as an isolated bilateral condition in which redundant bulbar conjunctival tissue interposes between the globe and the lower eyelid, and protrudes over the lid margin. He recognised that it causes tearing-a symptom of the watery eye-by mechanically disrupting the normal tear meniscus, and/or the flow of tears. He also stated that, unlike the chemotic conjunctiva of allergic conditions, it is not responsive to antihistamines or steroids. "Chalasis" is derived from the Greek, and refers to "relaxing" or "slackening," exemplified by bandages not tightly rolled around a limb.

It has been debated in the literature whether the dry eye syndrome may be associated with conjunctivochalasis. ${ }^{5-9}$ It has also been speculated that inflammation may be associated with conjunctivochalasis, since the adjacent lid margin and tarsal conjunctiva, which have been shown clinically to be inflamed, are closely related to the redundant bulbar conjunctiva. ${ }^{5}$ As Meller and Tseng ${ }^{5}$ point out, there have been no histopathological studies, controlled or otherwise, of conjunctivochalasis. Thus, we undertook this study in an attempt to define the histopathological and clinical characteristics of conjunctivochalasis in a watery eye group and an age matched control group.

\section{PATIENTS AND METHODS}

This was a prospective study of 24 controls and 18 patients (29 eyes). Patients underwent excision of redundant lid margin level bulbar conjunctiva (conjunctivochalasis) as complete or partial treatment of their watery eye symptomatology. Controls were matched for age. Controls had similarly sited normal conjunctiva removed at the conclusion of their standard endocapsular phacoemulsification procedure. ${ }^{10}$ Ethics committee permission was obtained from the South Eastern Sydney Area Health Service (Eastern section), and all controls underwent informed consent in keeping with the tenets of the Declaration of Helsinki. Standard surgical consent was obtained for all patients.

All patients were evaluated according to a standard watery eye protocol. Patients were examined for lid laxity and malposition, and on the slit lamp for conjunctivochalasis, punctal malposition, and punctal stenosis. The fluorescein dye disappearance test and Jones 1 and 2 testing were performed using $0.9 \%$ sterile saline. ${ }^{11}$ Nasal endoscopy with the $2.7 \mathrm{~mm}$ nasal endoscope was carried out in every patient. Schirmer's testing was done in eight of 18 patients. A clinical diagnosis as to the aetiology of the watery eye was made to the authors' satisfaction in every case.

The surgical technique employed for each group was as described by Hughes. ${ }^{2}$ Assisted local anaesthesia ${ }^{12}$ was used, with subconjunctival lidocaine with adrenaline being injected in each one of the patient group. Each member of the control group was undergoing routine cataract surgery using a retrobulbar or peribulbar anaesthetic. Their conjunctival specimen was removed at the conclusion of cataract surgery. For both groups, a surgical marking pen was used to outline the superior arm of the crescent along which the incision was made, tangential to and at the lower limbus. Then, drawing the freed, fornix based crescent of conjunctival tissue superiorly, an ellipse of conjunctiva was excised. We found the most effective method of closure was achieved with a running 10/0 nylon suture tied at each end of the wound; the

Abbreviations: DCR, dacryocystorhinostomy; FNLDO, functional nasolacrimal duct obstructions; PANDO, primary acquired nasolacrimal duct obstruction; VBT, Valsalva bubble test 
suture was removed at the slit lamp at 1 week. All specimens were sutured onto filter paper and fixed in $10 \%$ buffered neutral formalin. After more than 24 hours of fixation, the tissue was put through the automatic processor for histology. At least 15 sections were prepared from each specimen; three were stained with haematoxylin and eosin, three with periodic acid Schiff, three with Van Gieson elastic stain, and six remained unstained in case other stains were needed.

Statistical comparisons between clinical findings and pathological findings were made with Fisher's exact tests.

The average age of the control subjects was 69.4 years (range 59-85). There were eight male and 16 female controls. Control sections demonstrated normal conjunctival variation with non-keratinised stratified squamous epithelium, goblet cells, subepithelial collagen, elastic fibres, small blood and lymph vessels, leucocytes, histiocytes, and mast cells (fig l). A single control section of conjunctiva contained an abnormal, moderate, predominantly plasma cell, subepithelial infiltrate.

The average age of patients was 72.4 years (range 49-83). There were 12 male and six female patients. Histological sections demonstrated changes consistent with marked solar tissue damage in only one patient, who had bilateral marked conjunctival elastotic degenerative change (fig 2). A second had a lightly pigmented compound naevus with adjacent "elastosis" of the conjunctiva. Four patients had chronic nongranulomatous plasma cell and lymphocyte infiltration (fig 3) in the conjunctiva on one side. Of the four patients who had the inflammatory infiltrates, three had functional nasolacrimal duct obstructions (FNLDO) ${ }^{13}$ and one had a primary acquired nasolacrimal duct obstruction (PANDO). There was no statistically significant relation between inflammatory infiltrates and nasolacrimal obstruction, although the numbers were small $(p=0.107)$. Of the two patients who had elastosis, one had a FNLDO and the other had normal lacrimal drainage and was Jones 1 positive, and again there was no statistically significant association between the elastosis and nasolacrimal duct obstruction $(p=0.7)$. Pingueculae were not noted in any patients.

All 18 patients treated for conjunctivochalasis had watery eyes. Of the 18 patients, nine $(50.0 \%)$ had plerolacrima ${ }^{14}$ (tears filling the eyes but not running down the face), while seven $(38.9 \%)$ had epiphora (tears running down the face), and two patients $(11.1 \%)$ had both symptoms. In combination with conjunctivochalasis excision, nine patients $(50.0 \%)$ underwent lid surgery, five $(27.8 \%)$ underwent dacryocystorhinostomy (DCR) surgery, one (5.6\%) underwent both lid surgery and DCR, and four (22.4\%) underwent only conjunctivochalasis surgery.

Following DCR surgery, five of six patients had a positive Valsalva bubble test (VBT). ${ }^{15}$ Of the eight patients who underwent preoperative Schirmer's testing, only one suggested the presence of ocular surface drying.

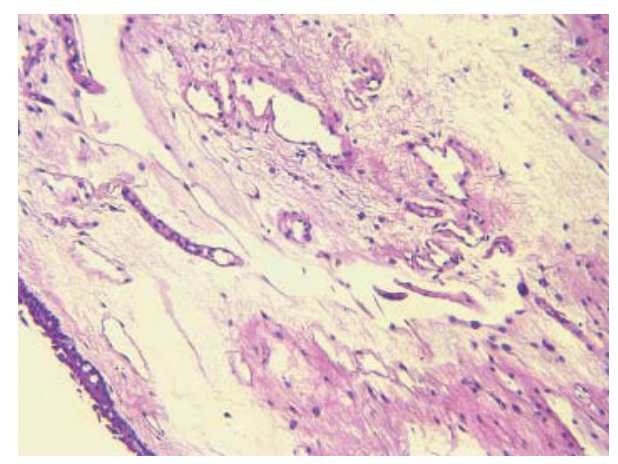

Figure 1 Normal conjunctiva ( $\times 10$, haematoxylin and eosin).

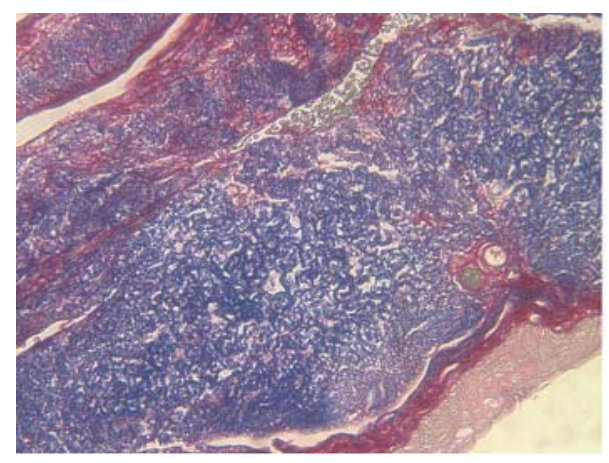

Figure 2 Marked elastotic degeneration of conjunctiva (20x, Van Gieson).

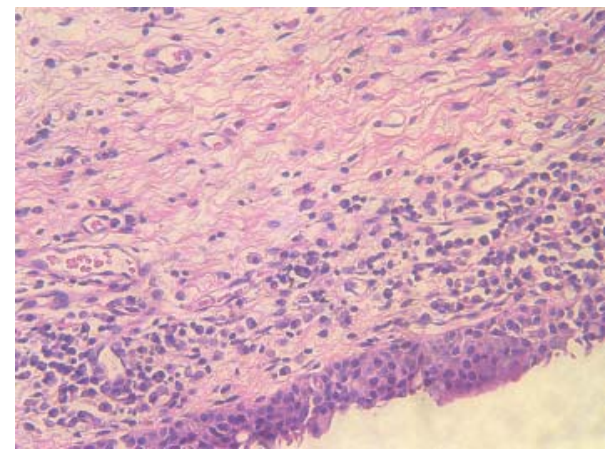

Figure 3 Subepithelial conjunctival infiltrate consisting mainly of plasma cells and lymphocytes (20x, haematoxylin and eosin).

Following surgery, 12 patients $(66.7 \%)$ were happy with their surgical result. Three patients $(16.7 \%)$ were substantially improved $(70 \%$ better). One patient $(5.6 \%)$ was not significantly improved and, despite being offered further surgery, did not return for follow up. One patient (5.6\%) is awaiting DCR surgery, and another $(5.6 \%)$ was lost to follow up.

Conjunctivochalasis is a significant and treatable cause of watery eyes. ${ }^{1-5}$ It is most commonly seen as an age related phenomenon in "rheumy" (Greek rheuma = stream) eyes, occurring typically in elderly people who have wet appearing eyes. Some investigators regard it as a manifestation of ocular surface drying. ${ }^{9}$ While we selected only eight patients to have Schirmer's testing, we found only one had any evidence of ocular surface drying. Moreover, all our patients presented not with dry eye symptomatology, but with watery eye symptomatology.

While it is not appropriate in this article to discuss the differential diagnosis ${ }^{16}$ of conjunctivochalasis, its aetiologies are protean. ${ }^{17}$ The clinician must keep in mind, according to Di Pascuale et al, ${ }^{9}$ that, in contrast with our group of watery eye patients, dry eyes are an important aetiology of conjunctivochalasis. Not only that, Di Pascuale's group delineated conjunctival staining, lid margin erosion, an obliterated tear meniscus, swelling of the punctum, and subconjunctival haemorrhage as central features of conjunctivochalasis, ${ }^{9}$ none of which were present in our patients. We thought this was probably because our patients had watery eyes as opposed to dry eyes.

Conjunctivochalasis has previously been described as "chronic localised conjunctival chemosis" by Kalin et al, in a group of seven patients where no other cause was found. ${ }^{16}$ Interestingly, in this group, histology on three of seven 
patients detected an inflammatory cellular infiltrate involving the conjunctiva. Two of seven patients had lymphangiectasia. ${ }^{16}$ As four of our 29 conjunctivochalasis specimens had a prominent inflammatory cell infiltrate, we thought it likely that the patients in Kalin et al's group demonstrated conjunctivochalasis, rather than a discrete new syndrome.

Regarding conjunctivochalasis, Li et al ${ }^{18}$ considered "existing pathologic data...scanty and conflicting." We sought to remedy that in our study, which examined 29 specimens from 18 patients with conjunctivochalasis, and 24 specimens from 24 control patients undergoing cataract surgery. ${ }^{10}$ In the week before our article was submitted, in May 2004, a clinicopathological study of conjunctivochalasis appeared in Cornea. ${ }^{19}$ In it, Watanabe et al, who beat us into print with a reasonably sized series, showed that in 44 cases who had both wet eye and dry eye symptomatology, there was only microscopic lymphangiectasia, and that in 39 cases. The major problem with their pathology is that dilated lymphatic vessels could have been an oedematous artefact in the subepithelial tissue of the conjunctiva, which may well not have been made to adhere to a support in the fixative. There were no inflammatory features seen. A further criticism of their study was that they removed the conjunctiva commencing $2 \mathrm{~mm}$ below the limbus and working inferiorly, thereby neatly ensuring that there would be no histopathological change in the tissue. The clinician can easily recognise this by inspecting the normal lower lid margin relation to the inferior limbus, the site of conjunctivochalasis. Put bluntly, conjunctivochalasis is found more superiorly than where Watanabe et al did their biopsies. Further, their control group consisted of only two patients, one aged 13 and the other 32, well out of the age range of patients with conjunctivochalasis.

In our study, 22 of 29 conjunctivochalasis specimens showed normal conjunctival histology. Histopathology in four eyes demonstrated a chronic inflammatory infiltrate. As noted above, this appearance has been previously described by Kalin et al in 1996. ${ }^{16}$ Nevertheless, its significance is at this point uncertain. It is possible that the patients of Kalin et al had keratoconjunctivitis sicca, and that the inflammatory response was simply a marker of drying rather than an initiating event. However, in our cohort, the patients were being actively managed for watery eyes. In Hoh's study, ${ }^{6}$ it was thought that keratoconjunctivitis sicca was responsible for the conjunctivochalasis of most of his patients, but no histology was carried out. Meller and Tseng ${ }^{5}$ felt that Hoh et $\mathrm{al}^{7}$ had not made a strong case for the association between conjunctivochalasis and dry eye. Nevertheless, Meller's and Tseng's main criticism was that an unstable tear film is not necessarily related to a potentially dry eye. By contrast, many clinicians think an unstable tear film is a definitive and major feature of ocular surface drying. ${ }^{20}$

It is possible that the conjunctival trauma produced by vigorous patient eye rubbing could cause conjunctivochalasis with an associated inflammatory reaction. Historically, none of our patients was an eye rubber. Perhaps the conjunctival stroma, already weakened by age, is disrupted further by blinking in an older person. Importantly, Li $e t a l^{18}$ have shown that increased expression of MMP-1 and MMP-3 is related to conjunctivochalasis. This work suggests that there may be a cytokine or some other stimulus that initiates the overexpression of the MMPs, resulting in an inflammatory response which may generate, at least in part, the development of conjunctivochalasis.

Elastosis was found in two patients (three specimens) in our cohort of 29. Li et al' $^{18}$ demonstration of the increased expression of MMP-1 and MMP-3 being greater in the conjunctivochalasis specimens may explain the development of conjunctival elastosis. This is supported by the rheumatological study by Toller and Wilcox, ${ }^{21}$ who suggested that elastotic change can occur as a "stress elastosis," as is seen in the temporomandibular joint. Thus, repeated eyelid blinking or rubbing could be the stimulus for the overexpression of MMPs. Also, the delayed tear clearance in watery eye patients may, as Meller et al suggest, lead to an accumulation of inflammatory cytokines in the tears, resulting in the upregulation of expression of MMP-1 and MMP-3, ${ }^{22}$ eventually leading to elastosis. Another hypothesised stimulus could be ultraviolet radiation, a speculation also shared by Li et $a l^{18}$ and supported by the work of Tulvatana et al..$^{23}$ We were surprised that only three of our specimens demonstrated elastosis, as we would have expected a significant ultraviolet effect in the production of this pathology, especially in sun exposed, elderly Australians.

The fact that the majority (22 of 29 specimens; $75.8 \%$ ) had normal conjunctiva, while only four specimens (13.8\%) had inflammatory changes and three specimens (10.3\%) demonstrated elastosis, suggests that the underlying aetiology and pathogenesis of conjunctivochalasis may be multifactorial. It is of interest that for the four patients with conjunctival inflammation, there was clinical evidence of nasolacrimal duct obstruction in all four (three patients had FNLDO and the other had PANDO). Again, the decreased tear clearance allows increased residence time for inflammatory mediators in the tears, contributing to the conjunctival inflammation. Thus, the delayed tear clearance described by Liu, ${ }^{4}$ may be related to the inflammatory conjunctival changes in conjunctivochalasis as described by Meller et al. ${ }^{22}$ On the other hand, it may be that the inflammation in the bulbar conjunctiva, and the inflammation presumably present in the lacrimal drainage system ${ }^{24}$ are of the same pathogenesis.

Ten of our 18 patients $(56.7 \%)$ had lid problems as the major aetiology of their watery eyes, and six of 18 patients $(33.3 \%)$ required definitive DCR surgery. We think this emphasises the multifactorial nature and complexity of the evaluation of many watery eye patients.

In conclusion, our study is the first to obtain both clinical and histological data comparing specimens of conjunctivochalasis from controls and patients in a watery eye cohort. We showed that seven of 29 specimens had pathological changes, as demonstrated by both inflammatory infiltrate and elastosis. Our understanding of the aetiology of conjunctivochalasis continues to develop, but local trauma, ultraviolet radiation, and the immunological effects of delayed tear clearance all appear at this stage to be contributory.

\section{ACKNOWLEDGEMENTS}

We thank Dr Murray Killingsworth for his assistance with photography.

We are indebted to Dr Astika Kappagoda, MBBS, PhD, for his translation of the Greek "chalasis."

\section{Authors' affiliations}

I C Francis, D G Chan, P Kim, G Wilcsek, M T Coroneo, The Ocular Plastics Unit, Department of Ophthalmology, The Prince of Wales Hospital, Randwick, Sydney, Australia

M Filipic, J Yong, The Department of Anatomical Pathology, South Western Area Pathology Service, Liverpool Hospital, Liverpool, Sydney. Australia

\section{REFERENCES}

1 Braunschweig P. Uber Faltenbildung der Conjunctiva bulbi. Klin Monatsb/ Augenheilkd 1921;66:123-4.

2 Hughes WL. Conjunctivochalasis. Am J Ophthalmol 1942;25:48-51.

3 Duke-Elder S. Conjunctival hyperplasia. System of ophthalmology. Vol XIII: The ocular adnexa. London: Kimpton, 1974:609.

4 Liu D. Conjunctivochalasis. A cause of tearing and its management. Ophthalmic Plast Reconstr Surg 1986;2:25-8. 
5 Meller D, Tseng SCG. Conjunctivochalasis: literature review and possible pathophysiology. Surv Ophthalmol 1998;43:225-32.

6 Hoh H. Stadium 4 der lidkantenparallelen conjunctivalen Falten-ein klinisches Zeichen des fortgeschrittenen Sicca-Syndroms. Contactologia 1999;21:121-30.

7 Hoh H, Schirra F, Kienecker C, et al. Lidparallele konjunktivale Falten (LIPCOF) sind ein sicheres diagnostisches Zeichen des trockenen Auges. Ophthalmologe 1995;92:802-13.

8 Grene RB. Conjunctival pleating and keratoconjunctivitis sicca. Cornea $1991 ; 10: 367-813$.

9 Di Pascuale MA, Espana EM, Kawakita T, et al. Clinical characteristics of conjunctivochalasis with or without aqueous tear deficiency. $\mathrm{Br} J$ Ophthalmol 2004;88:388-92.

$10 \mathrm{Ng} \mathrm{DT}$, Rowe NA, Francis IC, et al. Intraoperative complications of 1000 phacoemulsification procedures: A prospective study. J Cataract Refract Surg 1998;10:1390-5.

11 Hurwitz JJ. The lacrimal system. Philadelphia: Lippincott-Raven Publishers, 1996:52-3.

12 Francis IC, Schumacher RS, Haylen MJ. Assisted local anaesthesia for cataract surgery (ALACS). Aust NZ J Ophthalmol 1987;15:185-91.

13 Conway ST. Evaluation and management of "functional" nasolacrimal blockage: results of a survey of the American Society of Ophthalmic Plastic and Reconstructive surgery. Ophthal Plast Reconstr Surg 1994;10:185-7.

14 Francis IC. Canthus-sparing ectropion repair. Ophthalmic Plast Reconstr Surg 2002; 18:311-12.
15 Mulligan NB, Ross CA, Francis IC, et al. The Valsalva DCR Bubble Test: a new method of assessing lacrimal patency after DCR surgery. Ophthalmic Plast Reconstr Surg 1994;10:121-3.

16 Kalin NS, Orlin SE, Wulc AE, et al. Chronic localized conjunctival chemosis. Cornea 1996;15:295-300.

17 Yanoff M, Duker JS. Ophthalmology. 2nd ed. Philadelphia: Mosby, 2004:432.

18 Li D-Q, Meller D, Liu Y, et al. Overexpression of MMP-1 and MMP-3 cultured conjunctivochalasis fibroblasts. Invest Ophthalmol Vis Sci 2000;41:404-10.

19 Watanabe A, Yokoi N, Kinoshita S, et al. Clinicopathological study of conjunctivochalasis. Cornea 2004;23:294-8.

20 Sullivan DA, ed. Lacrimal gland, tear film, and dry eye syndromes: Basic science and clinical relevance. Ádv Exp Med Biol 1994;350:141-6.

21 Toller PA, Wilcox JH. Ultrastructure of the articular surface of the condyle in temporomandibular arthropathy. Oral Surg 1978;45:232-45.

22 Meller D, Li D-Q, Tseng SCG. Regulation of collagenase, stromelysin, and gelatinase $B$ in human conjunctival and conjunctivochalasis fibroblasts by interleukin-1b and tumor necrosis factor-a. Invest Ophthalmol Vis Sci 2000;41:2922-2929.

23 Tulvatana W, Bhattarakosol P, Sansopha L, et al. Risk factors for conjunctival squamous cell neoplasia: a matched case-control study. Br J Ophthalmol 2003;87:396-8.

24 Anderson NG, Wojno TH, Grossniklaus HE. Clinicopathologic findings from lacrimal sac biopsy specimens obtained during dacryocystorhinostomy. Ophthalmic Plast Reconstr Surg 2003;19:173-6. 